\title{
An Energy Balanced Algorithm for data communication in WSN
}

\author{
HAJAR LAGRAINI ${ }^{1}$, HICHAM OULDZIRA ${ }^{2}$,MOSTAFA CHHIBA ${ }^{1}$, \\ ABDELMOUMEN TABYAOUI $^{1}$, AHMED MOUHSEN $^{2}$ \\ 1 Radiations materials \& instrumentations Laboratory, Faculty of Sciences \& \\ Technology FSTS, University Hassan 1st. BP 577, 2600, SETTAT, MOROCCO \\ 2 Management, industrial \& innovation Laboratory, Faculty of Sciences \& \\ Technology FSTS, University Hassan 1st. BP 577, 2600, SETTAT, MOROCCO \\ Email: h.lagraini@uhp.ac.ma ,odzira@yahoo.fr, moschhiba@yahoo.fr, \\ atabyaoui@gmail.com, ahmed.mouhsen@uhp.ac.ma ,
}

\begin{abstract}
Wireless sensor networks are seeming as an emergent requisite for mankind. Such networks are still in research stage though, they have high prospective to be useful in practically every arena of life. A lot of study is done and lots of other is a waiting to be standardized. In this paper, clustering in WSNs is deliberate precisely. Furthermore, we improve one of the utmost popular WSN's routing algorithms "LEACH" as enhanced LEACH. Our improved algorithms, in comparison with basic algorithm overtake it by means of lifetime of cluster head and network lifespan. Subsequently, new thresholds are employed on improved algorithms that claim the performance even more. To finish a brief performance test of basic protocol, with ES-LEACH and ESD-LEACH is assumed bearing in mind energy leftover and distance metrics.
\end{abstract}

Keywords: sensor nodes, cluster routing, static sink, wireless sensor network.

\section{Introduction}

Data- data- data; we need instantaneous data in every feature of our lives. An automatic engineer desires to get data about his instrument running, a doctor needs to know about his patient's ECG interpretation, measured variations in earth and weather are required by geologists; list continue. To suffice this must, numerous networks are considered to transmit data. At first human to human, and then telephonic systems giving birth to WSs. Multi hop networks were intended to give additional autonomy of movement. And then arises the eon where machinemachine communication was announced. A treating device purchases data, process it and transmit it to another device. That data is auxiliary precised / gathered/ attached perceptively so that it can be accessible to us "humans". In WSNs, that gadget generally is named a sensor, or smart sensor node and has its own limits i.e. It is necessary proficient of sensing, treating and communicating. Each SN therefore need an electrical power source to ensure all these tasks. Taking into account applications of WSNs, fixing a battery on each SN is a the best option. Though, regulating consumption of power is one of the key tasks in these networks. These batteries need to be smart enough to give a SN extreme life regardless of being very small sized. Any technology that is in progress and expansion, provide lot of contests. Similarly WSNs do. Detecting, processing and communicating by very 
small sized nodes with power restraint is a pretentious thing. Consequently this is the main unease for scientist researchers. To enhance SN's lifespan, we must focalize on such techniques that can make maximum out of restricted battery. In any type of networks specifically WSN, for effective performance; these algorithms should be effectual. Many algorithms are presented that discourse limited battery issue in WSNs. In an effective WSN, we need efficient routing algorithm that has low-slung routing overhead and well-structured information collection tools to protect battery life of SN. Therefore clustering of the network is an alternative technique to promote power energy effectiveness. In hierarchical architecture sensing zone is divided to a numeral clusters and a set of nodes are haphazardly chosen as $(\mathrm{CH}) . \mathrm{CH}$ collects the data from non-CH members, united it and then transmit it to the BS for further treating [2]. Clustering thus distribute the energy load, reduce the energy ingestion and sustain the WSN life [3], [4], [5], [6]. In this paper an enhanced energy balanced algorithms are introduced for WSNs. our method proposes a new cluster head $(\mathrm{CH})$ selection technique based on tree elements: clustering, residual of energy and distance. This algorithm is an improvement of basic protocol presented in [7] and simulation result demonstrate that the enhanced protocol outperform LEACH in terms of network life time extension. This work is structured as follows. In Section 2 we discuss the literature review of existing algorithms. In section 3 we present clustering approach, $\mathrm{CH}$ selection and hierarchical routing algorithms. We further evaluated the proposal ES LEACH / ESD LEACH in section 4. To close, the conclusions are discussed in section 5 .

\section{Literature review:}

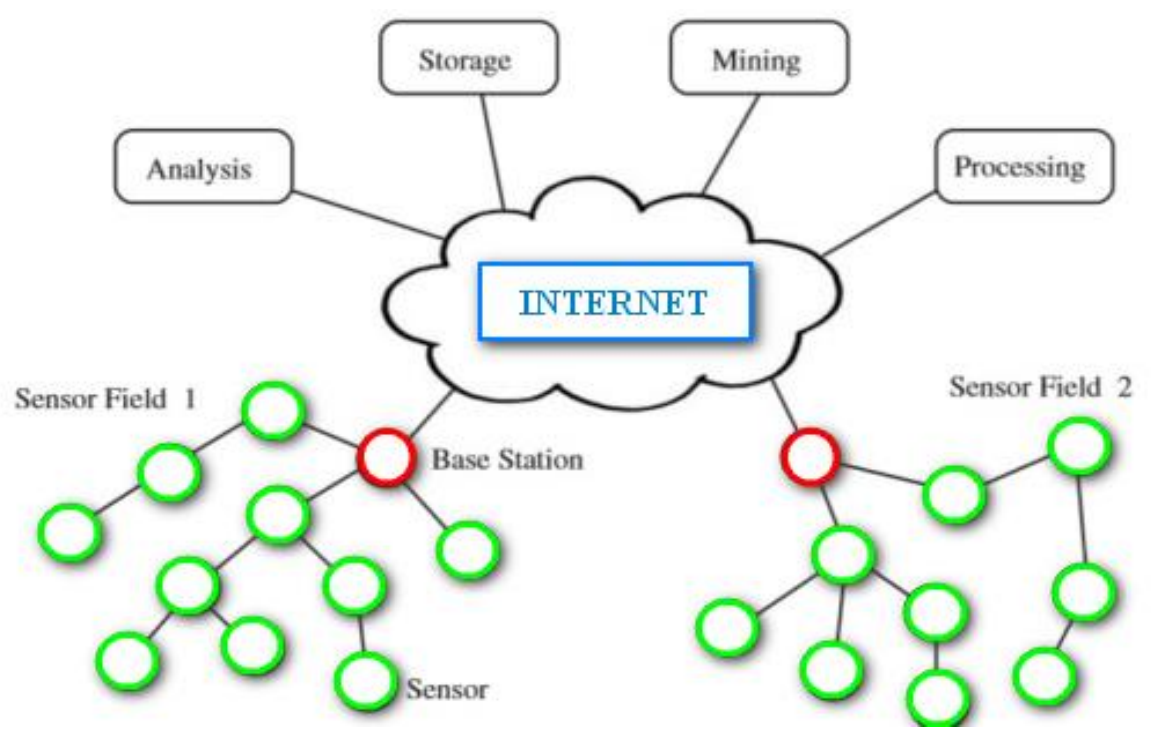

Fig 1: Overview of wireless sensor network with static sink

\subsection{CLUSTERING AND CLUSTER HEAD SELECTION}


In environmental sensing, clustering algorithms are easy and also effective. SNs are arbitrarily positioned in the monitoring area. Networks are clustered into distinct groups named clusters. Probabilities for SNs that are adjacent to each other are really elevated. Collection of information in clustering algorithms affords a power energy proficient technique for information aggregation. Correspondingly every cluster member node communicates a one-hop packet to the $\mathrm{CH}$; merely then the combined data-packet will be diffused to the sink by the selected $\mathrm{CH}$. whenever the collected information will go over information combination process just afterward that one data-packet will be created. The chosen $\mathrm{CH}$ will communicate the sensed information to the sink as an alternative of every SN distinctly. This process of arbitrarily turning the role of $\mathrm{CH}$ will regularly allocate the assigned work has been presented. To enhance the WSN lifetime the transmission capacity is necessary which is conceivable through using clustering technique. The entirely member-nodes transmit their information to the cluster heads owing to this battery is saved. Clustering approach allows proficient source distribution which primes to enhance conspiring of power source regulation. Any adjustment in the SN touches just that specific cluster and not the complete network.

\subsection{HIERARCHICAL CLUSTERING}

Similar to the alternative communication systems, one of the utmost imperative intentions characteristic of a WSN is extendibility. The gateway could become overburdened as the sensor substantiality rises adopting a one-hop transmission. Like time interval between the stimulation and response in communication is promising consequently overload and tracing of measures eventually becomes tough. One-hop network architecture is not ascendable if the WSN becomes expanded; it is not able to manage transmission. Clustering technique has been followed in a few routing methods to permit the network to deal with superfluous load and to envelope the large SN. The purpose for advancing hierarchical routing is to efficiently preserve the energy of the SNs by appealing them in multi-hop transceiving [25]. Packet gathering/combination is implemented in a WSN to reduce the amount of packets communicated to the BS. The $\mathrm{CH}$ choosing and cluster arrangement are founded on the residual of energy and the adjacency to the $\mathrm{CH}$ correspondingly. The principal aim of hierarchical routing technique is to conserve the energy ingesting of SNs. Establishing a cluster basically depend on level of residual or leftover of energy of SNs and adjacent to the cluster chief. This approach saves energy, based on clustering technique lifespan of the network is extended; scalability and energy ingesting are upgraded. Just one cluster head / cluster completes the routing mission and the rest of sensor members just transmit their information to the $\mathrm{CH}$. In vast WSNs, clustering approach shows a significant gain for the network it is smooth to administrate many clusters. Adopting clustering methods just preservation of the $\mathrm{CH}$ is sufficient. The SNs in wireless sensor networks are resource self-conscious which means they have restricted energy. Low energy adaptive clustering hierarchy is the most popular hierarchical routing algorithm in WSNs.

\subsection{PURE LEACH}

LEACH "Low-Energy Adaptive Clustering Hierarchy" is one of the most popular hierarchical routing algorithms. The function process of this protocol is subdivided into rounds. every round begins with a setup phase, pursued by steady state. In the first phase, every $\mathrm{SN}$ attempts to elite itself as a $\mathrm{CH}$ conforming to probability model. For choosing a $\mathrm{CH}$, every $\mathrm{SN}$ set up a arbitrary number in between zero and one. In case that the number is inferior than the threshold T (n), the SN choose itself as a $\mathrm{CH}$ for prevailing round. The threshold $\mathrm{T}(\mathrm{n})$ is calculated thusly:

$$
T(n)= \begin{cases}\frac{P}{1-P *\left(r \bmod \frac{1}{P}\right)} & ; \text { if } n \in G \\ 0 & ; \text { otherwise }\end{cases}
$$

Where: $\mathrm{p}$ is the desired percentage of cluster heads in the sensor area and $\mathrm{r}$ is the prevailing round number, where $\mathrm{G}$ is the set of SNs that have not been chosen as cluster heads in the last $1 / \mathrm{p}$ rounds [2][8]. 


\section{PROPOSED ALGORITHM}

On examining the properties of LEACH, the SNs chosen as CHs are not an ultimate choice for communicating the information to the base station. In the interest to unravel this issue, this fragment gives an effective protocol to provide a considerable saving in battery ingestion. The suggested protocols are suitable for WSN; which has following qualifications: SNs are static, i.e., once they are randomly diffused, they remain in the same position. Every SN has a special ID and recognizes its location and it's residual of energy. Moreover, every SN has enough energy to communicate with the sink. The entire sensor nodes have the similar incipient power (Einit) and their power sources are not rechargeable or exchangeable. Nodes expire with the expiration of their battery. In our proposition, the possibility or chance for a SN to be cluster head is decided by its distance to the base station and by energy leftover of sensor node. though diminishing of broadcasting energy reduces exponentially with the augmenting diffusion range in wireless transmission. The level of remaining energy of SNs and the range between $\mathrm{CHs}$ and the sink are two indispensable metrics that possess an influence on total energy ingestion in every round. accordingly, we will have to consider the selection of the cluster head in order to distance of cluster heads to the BS and range of CMs to the $\mathrm{CH}$ are minimal, which will considerably enhance energy depletion for wireless sensor networks lifetime optimization. In our proposed protocol a $\mathrm{SN}$ could be chosen as $\mathrm{CH}$ basically on two metrics energy of the sensor and its distance from sink, the threshold value is calculated as follow:

$$
\begin{aligned}
& \mathrm{T}(\mathrm{n})=\left\{\begin{array}{cc}
\mathrm{C} * \frac{\mathrm{E}_{\mathrm{curr}}}{\mathrm{E}_{\text {init }}}+\mathrm{b} * \mathrm{p} *\left[\frac{\mathrm{D}_{\max }-\mathrm{D}}{\mathrm{D}_{\max }-\mathrm{D}}\right]^{2} ; & \text { if } \mathrm{n} \in \mathrm{G} \\
0 & ; \text { otherwise }
\end{array}\right. \\
& \text { While; } \mathrm{C}=\frac{P}{1-P *\left(r \bmod \frac{1}{P}\right)}
\end{aligned}
$$

Where, $\mathrm{E}_{\text {curr }}$ is the energy leftover of the $\mathrm{SN}$ for the present round and $\mathrm{E}_{\text {init }}$ is the primary energy , on suggested protocol, cluster head is chosed based on range of SN and sink. The SN which is close to the BS will be chosen as the $\mathrm{CH}$. This enhancement takes remaining energy and distance into account, further it considers the distance from node to cluster head BS and compares the distance from node $\mathrm{CH}$ and $\mathrm{BS}$. $\mathrm{D}_{\max }$ : Represents the maximum distance from SN to BS. D represents distance from sensor node to the BS. Based on left over of energy and distance from BS, threshold criteria $\mathrm{T}(\mathrm{n})$ is calculated.

\section{RESULTS AND SIMULATION}

The simulation results are provided of the proposed protocoles performed in which the Sink is fixed in MATLAB2017a simulator and compared with the results of the basic protocol. Comparison is done in the number of alive nodes, energy of the whole network and the number of received messages to the sink. Table 1 shows simulation parameters used in this work.

Table 1 : Simulation Environment Specifications

\begin{tabular}{ll}
\hline Parameter & Value \\
\hline Simulator & Matlab2017a \\
WSN area / m2 & $200 * 200$ \\
Number of nodes & 160 \\
Number of rounds & 5000 \\
Initial energy of node : Ei & 0.5 Joules
\end{tabular}


Desired percentage of $\mathrm{CHs}: \mathrm{P}$

Transmitter Amplifier Energy $\left\{\begin{array}{c}\text { Emp } \\ \text { Efs }\end{array}\right.$

Data Aggregation Energy Eagg

Electric energy
0.05

$0.0013 \mathrm{pJ} / \mathrm{bit} / \mathrm{m} 4$

$10 \mathrm{pJ} / \mathrm{bit} / \mathrm{m} 2$

$5 \mathrm{~nJ} / \mathrm{bit}$

$50 \mathrm{~nJ} / \mathrm{bit}$

ESD-LEACH

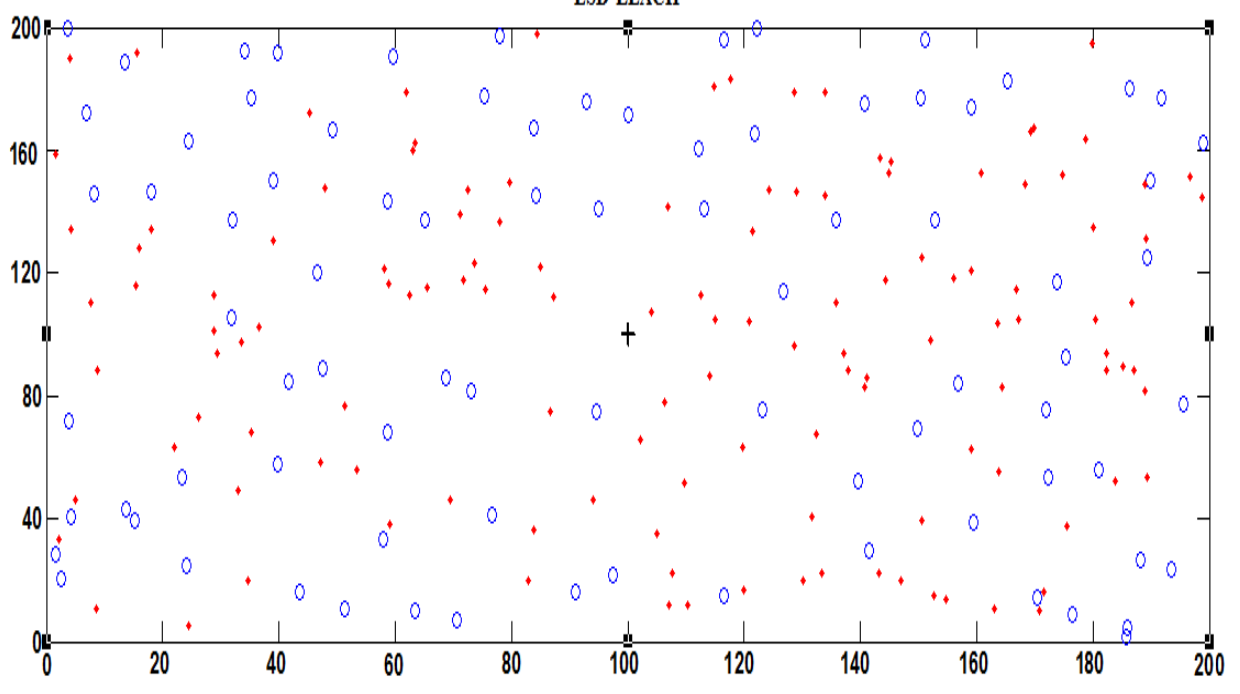

Fig. 2. alive node vs dead nodes in 5000 rounds using the enhanced algorithm

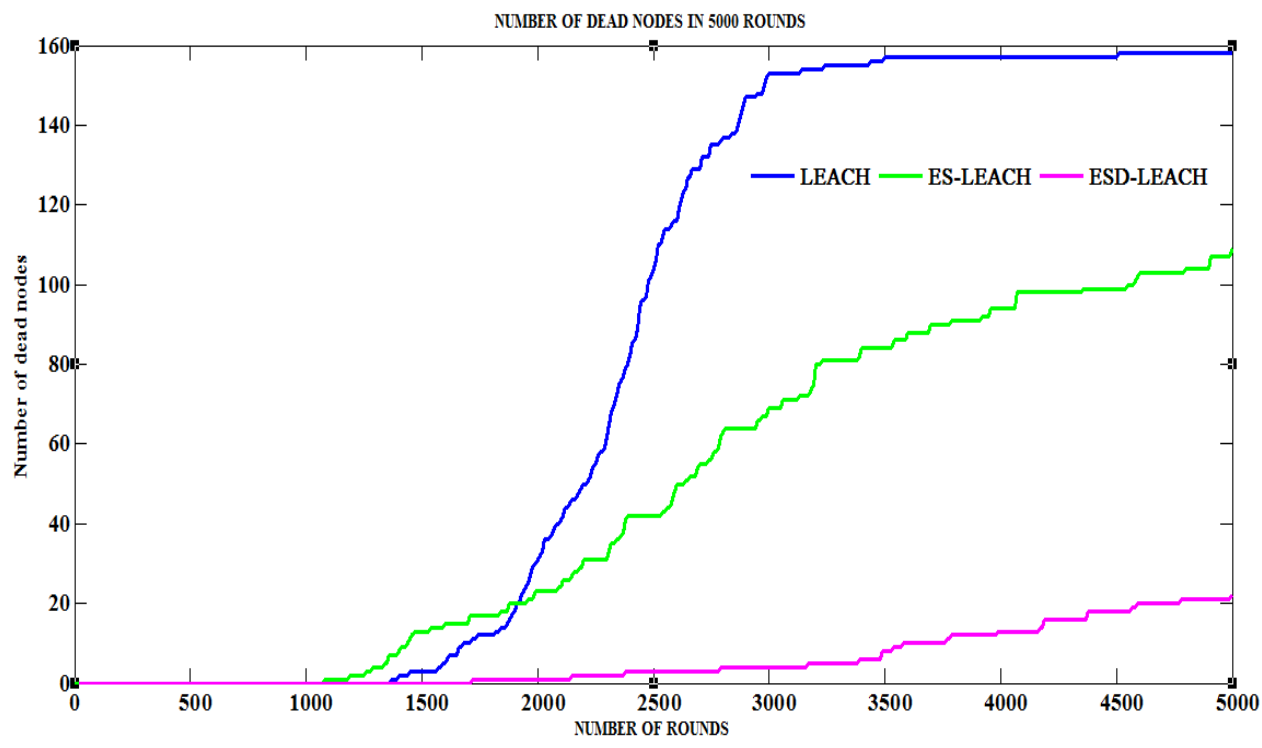

Fig. 3: Dead Nodes over rounds 


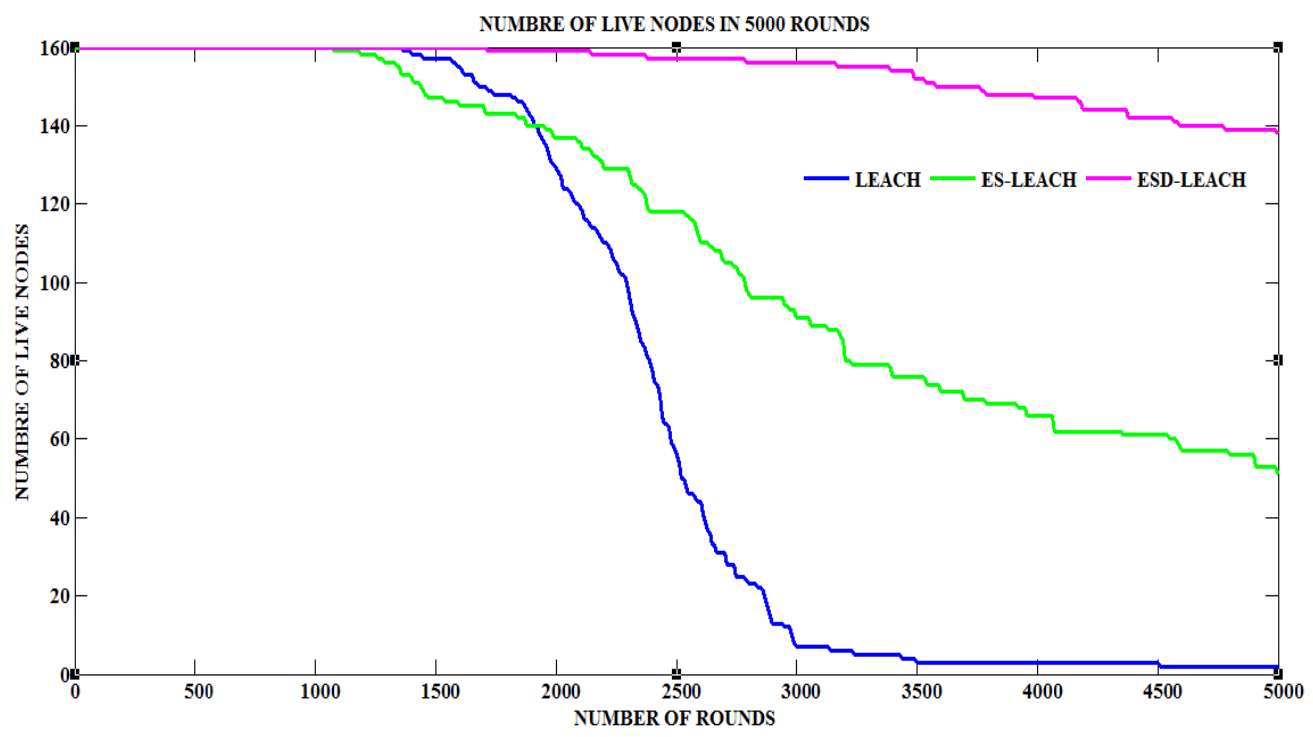

Fig. 4 Alive nodes over time in 5000 rounds

Figures 3, 4 show the comparison of pure LEACH, ES-LEACH and ESD LEACH algorithms in terms of amount of alive sensors in each round through all network life time of the 3 algorithms for 160 nodes diffusion in $200 \times 200$ areas.

The results are shown in Figure 3; SNs in ESD LEACH have a longer network battery life than the pure LEACH. The reduction in power ingesting of ESD LEACH is done by comparing it with that of the pure algorithm. We observe from the figure that the nodes of basic algorithm start to die approximately at 1600 rounds. However, ESD LEACH SNs start to die at 3500rounds. We can also clearly notice that the network lifetime of proposed algorithm prolonged until 5000 rounds, whereas that of pure LEACH ended at 3500 rounds.

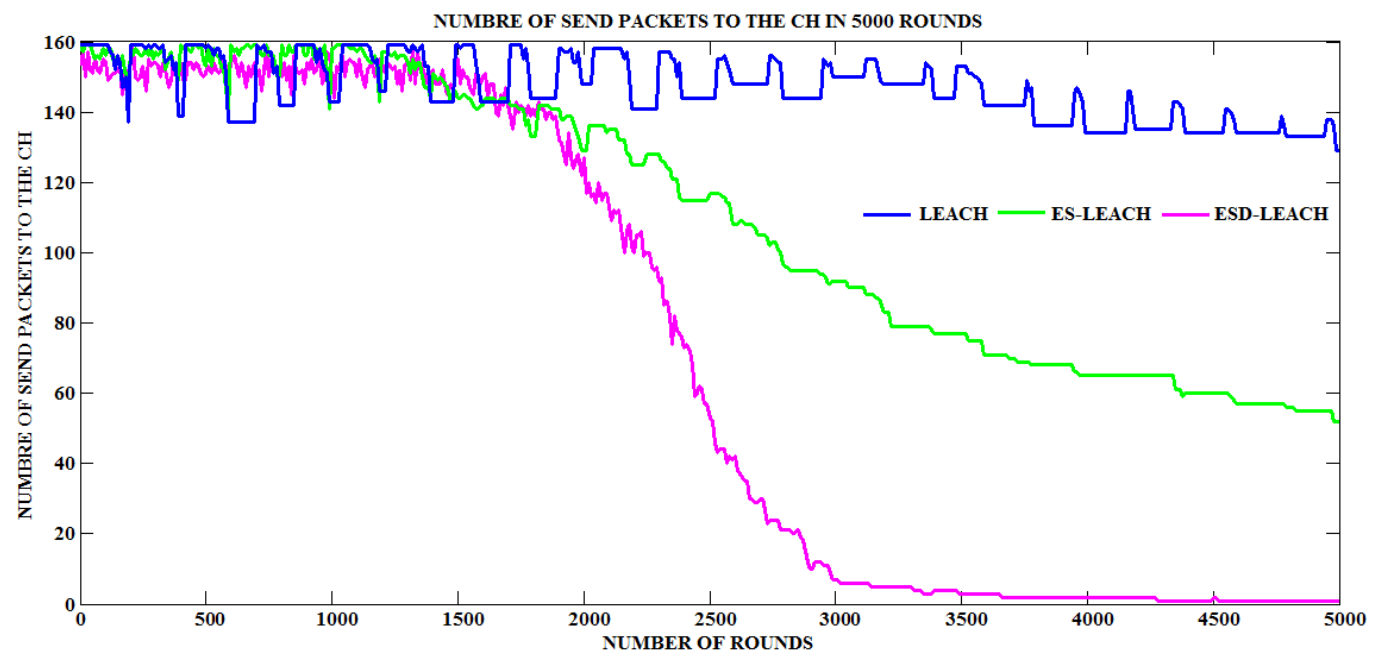

Fig. 5 : number of send packets to the $\mathrm{CH}$ in $200 \times 200 \mathrm{~m}^{2}$ 


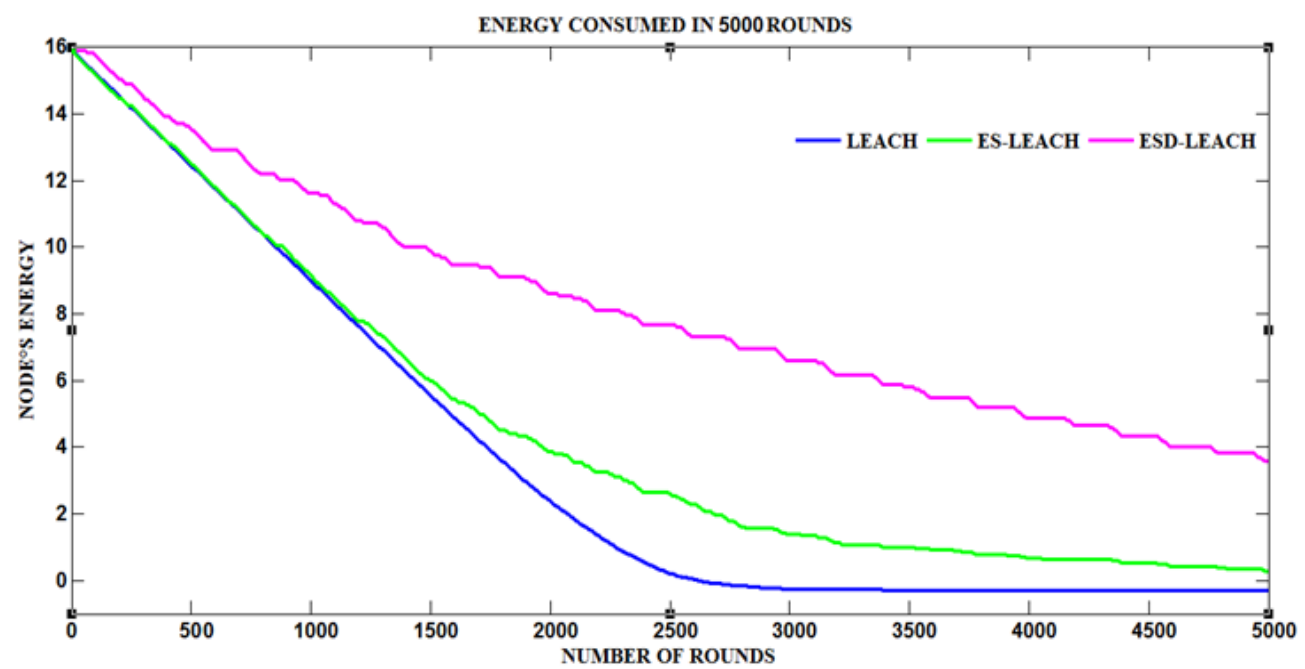

Fig.6: the comparison of energy ingestion rate in 5000 rounds.

We focus on the complete left over energy of SNs. Figure 6 shows the diminishing of this energy/ round. When comparing our algorithms to the basic algorithm, it's crystal clear that in our modified protocols, remaining energy diminishes leisurelier than basic one. In other words, our algorithms are proficient to balance energy ingesting much greater than pure algorithm. This verifies that we have picked an effectual way for choosing cluster heads based on outstanding energy level and the range between the SN and the SINK.

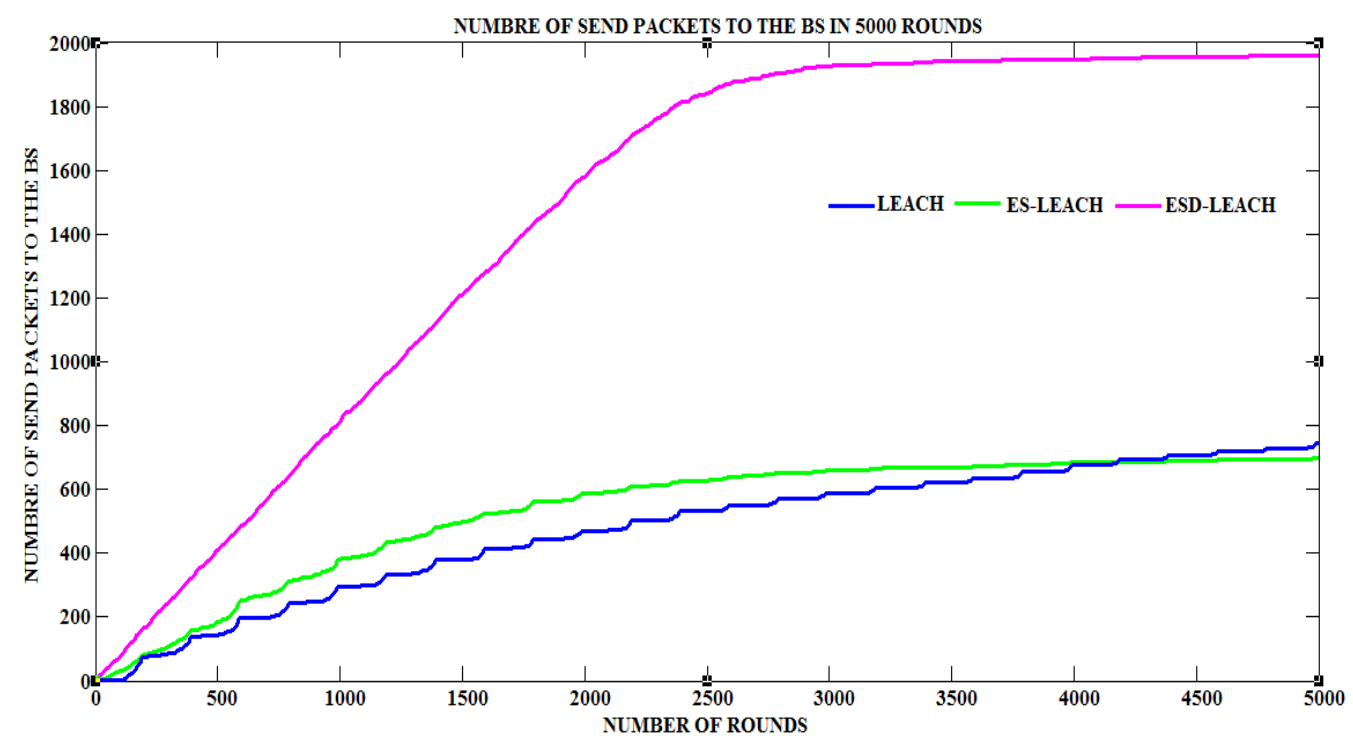

Fig.7 : Average number packets that received to the sink. 
This Figure 7 shows delivery of data packets to the BS. ES LEACH , ESD LEACH packet transmission give better rate then pure LEACH.

\section{CONCLUSIONS AND FUTUR WORK}

In this paper, we proposed an energy balanced algorithm for wsn which is an enhancement to pure $\mathrm{LEACH}$ protocol, we presented a new technique to choose the $\mathrm{CHs}$ in each round which basically depends on two metrics distance and energy level od nodes. In ES LEACH / ESD LEACH, we think closest $\mathrm{CH}$ to the sink will consumes fewer energy than other nodes since the information transmission dissipates the ultimate energy in WSNs. Simulation graphs confirmed that the enhanced ESD LEACH surpass basic LEACH in stretching the networks life. present results in this work can achieve performance improvements, there are other areas for improvement to make our ESD LEACH best suited everywhere. As the smart sensor nodes have limited power energy, therefore the nodes die after a certain period limit. Future work directions are to take the nodes themselves as solar aware nodes which recapture energy it selves, so that our protocol will be more energy sufficient. And another provision is to make our algorithm in to hierarchal protocol by forming "TOP clusters" out of the cluster head nodes and TOP clusters will process all the information from the CHs. Therefore the energy dissipation of every $\mathrm{CH}$ will be condensed to transfer data to the sink. This enhancement will make the ESD LEACH effective for an extensive range of smart networks.

6

References

[1] Akyildiz, I. F., Su, W., Sankarasubramaniam, Y., \& Cayirci, E. (2002). Wireless sensor networks: a survey. Computer networks, 38(4), 393-422.

[2] Akkaya, K., \& Younis, M. (2005). A survey on routing protocols for wireless sensor networks. Ad hoc networks, 3(3), 325-349.

[3] Al-Karaki, J. N., \& Kamal, A. E. (2004). Routing techniques in wireless sensor networks: a survey. IEEE wireless communications, 11(6), 6-28.

[4] Liang, C. K., Huang, Y. J., \& Lin, J. D. (2008, March). An energy efficient routing scheme in wireless sensor networks. In Advanced Information Networking and Applications-Workshops, 2008. AINAW 2008. 22nd International Conference on (pp. 916-921). IEEE.

[5] Zheng, J., \& Jamalipour, A. (2009). Wireless sensor networks: a networking perspective. John Wiley \& Sons.

[6] Lindsey, S., \& Raghavendra, C. S. (2002). PEGASIS: Power-efficient gathering in sensor information systems. In Aerospace conference proceedings, 2002. IEEE (Vol. 3, pp. 3-3). IEEE.

[7] Heinzelman, W. B., Chandrakasan, A. P., \& Balakrishnan, H. (2002). An application-specific protocol architecture for wireless microsensor networks. IEEE Transactions on wireless communications, 1(4), 660-670.

[8] Bian, Q., Zhang, Y., \& Zhao, Y. (2010, May). Research on clustering routing algorithms in wireless sensor networks. In Intelligent Computation Technology and Automation (ICICTA), 2010 International Conference on (Vol. 2, pp. 1110-1113). IEEE.

[9] Long-long, X., \& Jian-jun, Z. (2010, August). Improved LEACH cluster head multi-hops algorithm in wireless sensor networks. In Distributed Computing and Applications to Business Engineering and Science (DCABES), 2010 Ninth International Symposium on(pp. 263-267). IEEE.

[10] Hou, R., Ren, W., \& Zhang, Y. (2009, October). A wireless sensor network clustering algorithm based on energy and distance. In Computer Science and 
Engineering, 2009. WCSE'09. Second International Workshop on (Vol. 1, pp. 439-442). IEEE.

[11] Ali, M. S., Dey, T., \& Biswas, R. (2008, December). ALEACH: Advanced LEACH routing protocol for wireless microsensor networks. In Electrical and Computer Engineering, 2008. ICECE 2008. International Conference on (pp. 909-914). IEEE.

[12] Chen, G., Zhang, X., Yu, J., \& Wang, M. (2012, July). An improved LEACH algorithm based on heterogeneous energy of nodes in wireless sensor networks. In Computing, Measurement, Control and Sensor Network (CMCSN), 2012 International Conference on (pp. 101-104). IEEE.

[13] Ahmad, A., Latif, K., Javaidl, N., Khan, Z. A., \& Qasim, U. (2013, May). Density controlled divide-and-rule scheme for energy efficient routing in Wireless Sensor Networks. In Electrical and Computer Engineering (CCECE), 2013 26th Annual IEEE Canadian Conference on (pp. 1-4). IEEE.

[14] Bakaraniya, P., \& Mehta, S. (2013). K-leach: An improved leach protocol for lifetime improvement in wsn. International Journal of Engineering Trends and Technology, 4(5), 1521-1526. 2013. 